\title{
Effects of Filler Wire Intervention on Gas Tungsten Arc: Part II - Dynamic Behaviors of Liquid Droplets
}

\section{A novel sensing method for monitoring and controlling weld quality was tested by studying droplet behavior and its effect on the arc}

BY S. ZOU, Z. WANG, S. HU, G. ZHAO, W. WANG, AND Y. CHEN

\begin{abstract}
In gas tungsten arc welding (GTAW), the filler wire increases the deposition efficiency and influences the welding stability. Its interactions with the gas tungsten arc (GTA) are significant to better understand the welding process and to monitor and control weld quality. In view of this, the first part of the work, Effects of Filler Wire Intervention on Gas Tungsten Arc: Part I - Mechanism, explained the interaction mechanisms between the filler wire and the gas tungsten arc based on the proposed arc-sensing method of detecting probe voltage (i.e., the voltage signal between the filler wire and the tungsten electrode/workpiece). In this second part of the work, experiments were designed to make the filler wire melt in different areas of the arc to study the dynamic behaviors of the droplet and its effect on the arc. Typical metal transfer modes are discussed, and droplet oscillation is geometrically characterized through image processing and then analyzed in the time domain and time-frequency domain. The results show that the liquid droplet affects the arc through its transfer to the weld pool, its oscillation, and occupying the arc space. Information about these dynamic behaviors can be easily reflected in the probe voltage, which would be a valuable signal to monitor the process stability in GTAW with filler wire. This work shows the potential of the proposed sensing method for monitoring and controlling weld quality in all welding positions, GTA-based additive manufacturing, etc.
\end{abstract}

\section{KEYWORDS}

- Arc Voltage - Droplet Oscillation • Metal Transfer

- Filler Wire • Arc Sensing • Gas Tungsten Arc Welding (GTAW)

\section{Introduction}

Gas tungsten arc welding (GTAW), a conventional metal fabrication technique, has been widely used in various industrial fields to produce high value-added products. Under the traction of information technology, it is a recent trend to further improve the automation and intelligence of this technique. It is well known that the stability of metal fabrication with electric arc as the heat source is affected by many factors under harsh working conditions, and the traditional open-loop, constant-parameter automated GTAW usually does not possess intelligent perception, nor can it correct the welding process in real time to make it advance toward the ideal direction. Therefore, real-time monitoring and controlling of the welding process is significant to ensure weld quality.

A feasible proposal for the monitoring and controlling of weld quality is often based on some particular understanding of the welding process. Prior to putting forth such a proposal, it is critical to mine the characteristic information directly or indirectly related to the state of the welding process based on a specific sensing method. This information may provide useful clues or can be used as the input for feedback control of weld quality. Over the years, physical information accompanying the welding process, such as spectral (Ref. 1), infrared thermal (Ref. 2), acoustic (Ref. 3), and electrical (Ref. 4), has received extensive academic attention. Researchers have also obtained some valuable information by virtue of the physical properties inherent in the arc welding process, such as analyzing the dynamic behaviors of the weld pool based on the principle of liquid surface specular reflection (Ref. 5).

Although diverse potential information and mechanisms have been found, researchers have devoted more attention to the weld pool status than the influence of the liquid metal generated from the filler wire on the welding process. For GTAW, the employment of filler wire can not only form a reasonable convex shape on both sides of the weld bead, but it can also improve the weld strength and help to optimize the weld composition with the addition of diverse alloying elements. However, as part of the welding process, the filler wire tends to increase the instability of the process, thus affecting the weld quality. Given all of this information, the goal of this work is to analyze the effects of filler wire intervention on the gas tungsten arc (GTA). To this end, an innovative but simple sensing method of detecting the probe voltage (i.e., the voltage signal between the filler wire and tungsten electrode/workpiece) was proposed to help the analysis. This method is expected to have potential for the condition monitoring of the welding process. 

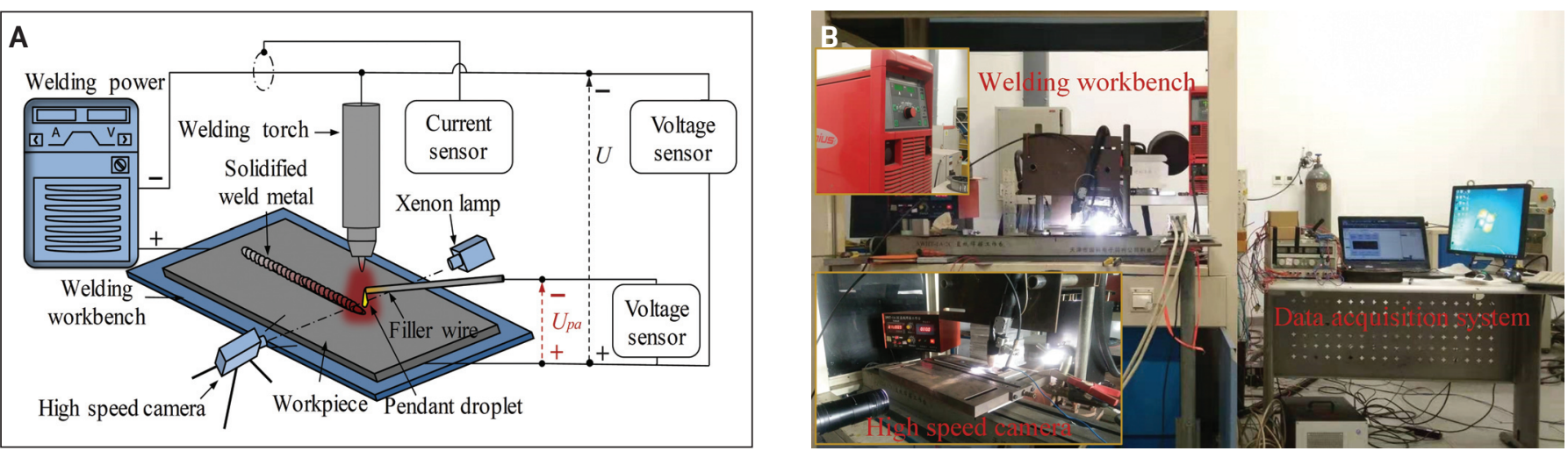

Fig. 1-Experimental system: A - Schematic diagram; B - actual system.

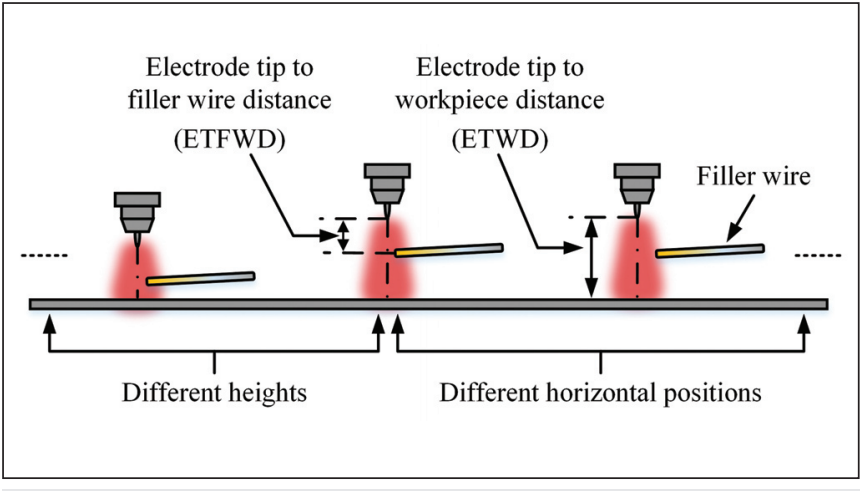

Fig. 2 - Schematic diagram of the experimental design.

In the first part of this work (Ref. 6), the interaction mechanisms between the GTA and the filler wire as a metal conductor were clarified, where a tungsten probe was employed to replace the filler wire and to interact with the arc, while the dynamic behaviors of the liquid metal generated at the end of the filler wire were avoided. Therefore, in this second part of the work, the metal transfer and the behavior of pendant droplets, as well as their effects on the arc, are thoroughly discussed. This work helps to understand the dynamic behaviors of the liquid droplet and paves the way for real-time control of weld quality based on the proposed sensing method.

\section{Experimental Setup and Design}

\section{Experimental Setup}

Figure 1 shows the experimental system. As presented in
Fig. 1A, the overall system was composed of the following parts: a welding system consisting of a Fronius MagicWave 4000 GTAW power source (constant-current mode), welding torch, a CK Worldwide WF-3 wire feeder, and SWHT-1A-2C welding workbench; a visual sensing system, including an Acuteye high-speed camera and a xenon lamp used to illuminate the field of interest; and an electrical signal acquisition system, where data acquisition was achieved by Hall sensors, USB-4711A data acquisition card, and computer MATLAB ${ }^{\circledR}$ programming. The actual overall system is shown in Fig. 1B.

\section{Experimental Design}

The workpieces were Q235 mild steel plates with a dimension of $300 \times 60 \times 4 \mathrm{~mm}$. ER70S-6 filler wire with a diameter of $1.2 \mathrm{~mm}$ was used as filler material. Pure argon of $99.99 \%$ was used as shielding gas with a flow rate of 10 $\mathrm{L} / \mathrm{min}$. The tungsten electrode was $2.4 \mathrm{~mm}$ in diameter and was placed with a protruding length of $5 \mathrm{~mm}$ from the nozzle. Bead-on-plate welding was done in the flat position.

As illustrated in Fig. 2, the experiments designed in this part of the work had the end of the filler wire melt in different spatial areas of the arc column to study the dynamic behaviors of the liquid metal generated at the end of the filler wire. In all experiments from \#1 to \#5, filler wire was employed and fed from the front of the weld pool, and the ratio of the welding speed $(v)$ to wire feed speed $\left(v_{f}\right)$ was constant. The height of the filler wire was changed by adjusting the electrode tip to workpiece distance (ETWD) so that its end could be melted at different heights and the filler wire could also be melted at different horizontal positions by changing $v_{f}$. The specific experimental parameters

Table 1 - Experimental Parameters

\begin{tabular}{|c|c|c|c|c|c|}
\hline Experiment & $I(A)$ & $v(\mathrm{~cm} / \mathrm{min})$ & ETWD $^{*}(\mathrm{~mm})$ & $\mathrm{ETFWD}^{* \star}(\mathrm{mm})$ & $v_{f}(\mathrm{~cm} / \mathrm{min})$ \\
\hline$\# 1$ & 150 & 5.25 & 5.5 & 3 & 76.2 \\
\hline \#2 & 150 & 5.25 & 8.5 & 3 & 76.2 \\
\hline \#3 & 150 & 3.5 & 7.5 & 3 & 50.8 \\
\hline \#5 & 150 & 7 & 7.5 & 3 & 101.6 \\
\hline
\end{tabular}

*Note: ETWD means electrode tip to workpiece distance; **Note: ETFWD means electrode tip to filler wire distance. 


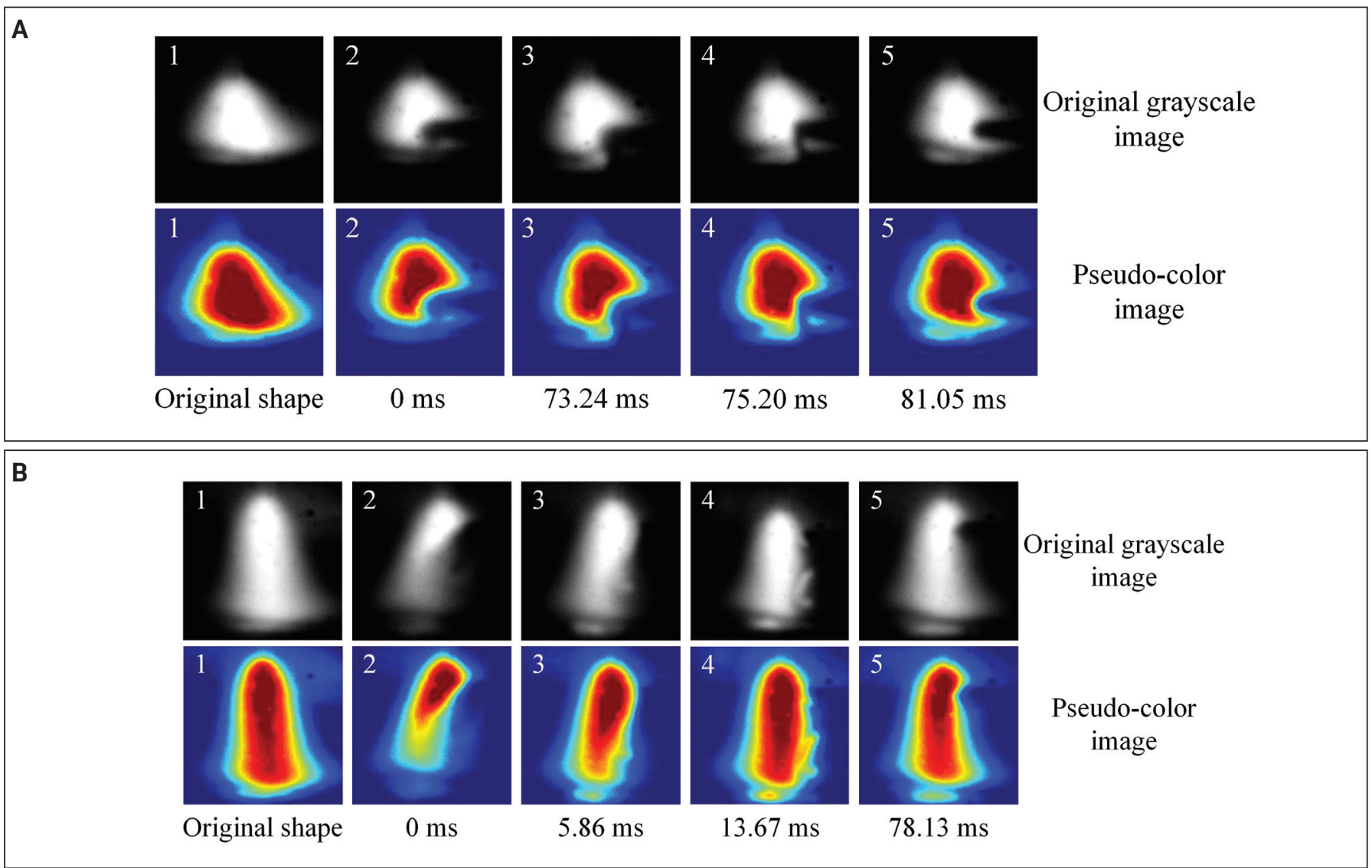

Fig. 3 -Arc shapes of GTAW with filler wire: $A-B$ ridging transfer (ETWD $=6 \mathrm{~mm}) ; B-$ free-flight transfer $(E T W D=9 \mathrm{~mm})$.

are shown in Table 1 , and these experiments were conducted with the welding torch in the stationary position while the welding workbench was moving.

As in the first part of the work (Ref. 6), the probe voltage $\left(U_{p a}\right.$, i.e., the voltage between the filler wire and the workpiece) and the arc voltage ( $U$, i.e., the voltage between the tungsten electrode and the workpiece) were collected simultaneously at a frequency of $1024 \mathrm{~Hz}$. The electrical signal data was then filtered by a $12^{\text {th }}$-order Butterworth low-pass filter with a passband frequency of $100 \mathrm{~Hz}$ and a $40 \mathrm{~dB}$ attenuation at a cutoff frequency of $150 \mathrm{~Hz}$. In addition, the measured welding current was used to ensure that the voltage fluctuations were not caused by the current fluctuations.

\section{Results and Discussion}

\section{Metal Transfer}

In GTAW with filler wire, the metal transfer modes were generally divided into bridging transfer and free-flight transfer. During the bridging transfer, a short-circuit liquid bridge was formed between the end of the filler wire and the weld pool. While in free-flight transfer, the liquid droplet could not immediately contact the weld pool after it left the end of the filler wire. Apparently, the metal transfer mode is related to the distance of the filler wire from the weld pool. Figure 3 shows the arc shapes in these two typical metal transfer modes. After the filler wire was inserted into the arc column, the arc shape was indeed disturbed to various degrees. As the melting of the filler wire generated a more dynamic liquid droplet, the arc shape also changed dynamically. Similar to the effect of the metal probe on the arc shape in the first part of this work (Ref. 6), the deflection of the arc shape and its cross-section change can also be observed after the filler wire was introduced.

For the interrupted bridging transfer in Fig. 3A, when there was a liquid bridge between the weld pool and the filler wire (at 73.24 and $75.20 \mathrm{~ms}$ ), the arc was attracted by the filler wire, otherwise the arc would be repelled by the filler wire (at 0 and $81.05 \mathrm{~ms}$ ). This can be judged by the degree of deflection on the left side of the arc. For the freeflight transfer in Fig. 3B, the filler wire repelled the arc the entire time due to the absence of the liquid bridge. This is because the liquid bridge integrated the filler wire and the weld pool, so the filler wire was no longer suspended alone in the arc column but became part of the anode, which changed the main conductive path of the arc.

Critical metal transfer modes were realized in Experiments \#1 and \#2, and the corresponding electrical signals are respectively shown in Figs. 4 and 5. In Fig. 4, the uninterrupted bridging transfer was maintained for a period of time as shown in I1. $U$ was relatively stable, and $U_{p a}$ was nearly zero due to the short circuiting between the weld pool and filler wire. During this time, the filler wire had stable effects on the arc just like a static metal probe, while also attracting the arc. However, the voltage signal was not as 


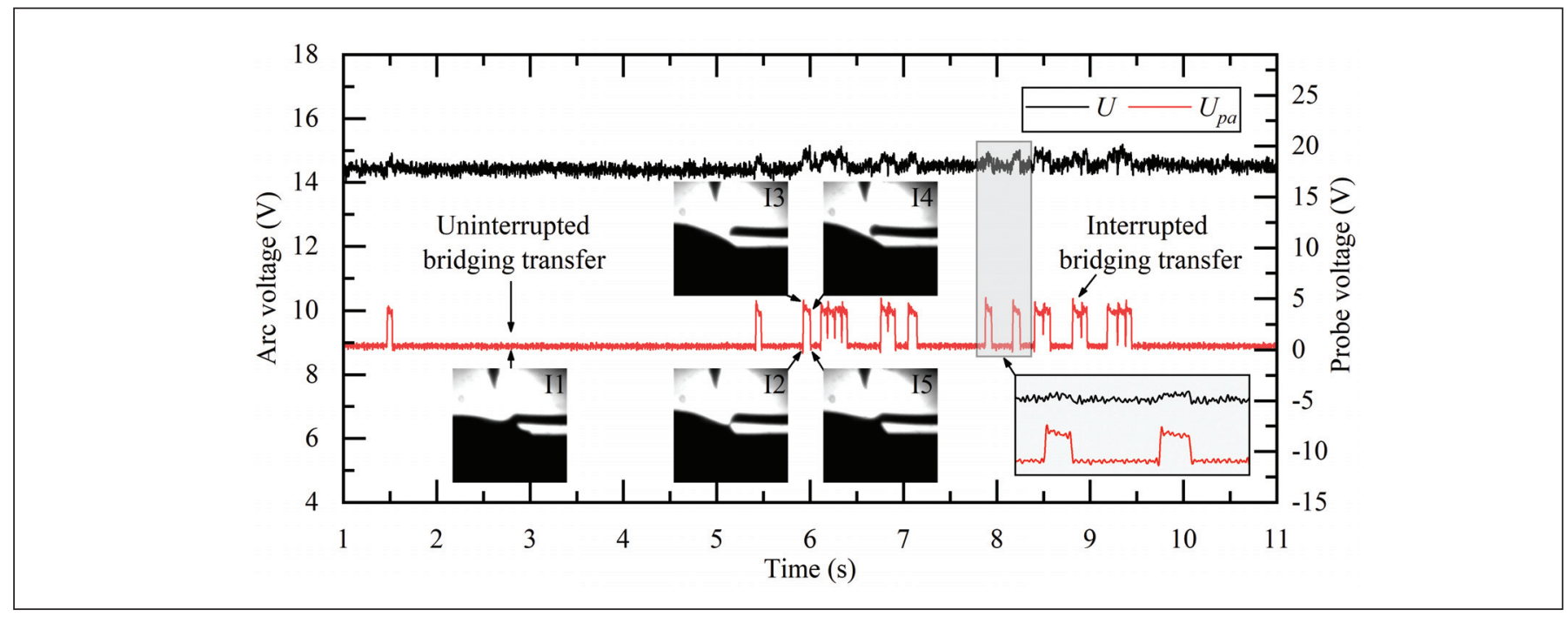

Fig. 4 - Electrical signals in Experiment \#l (ETWD = $5.5 \mathrm{~mm}$ ).

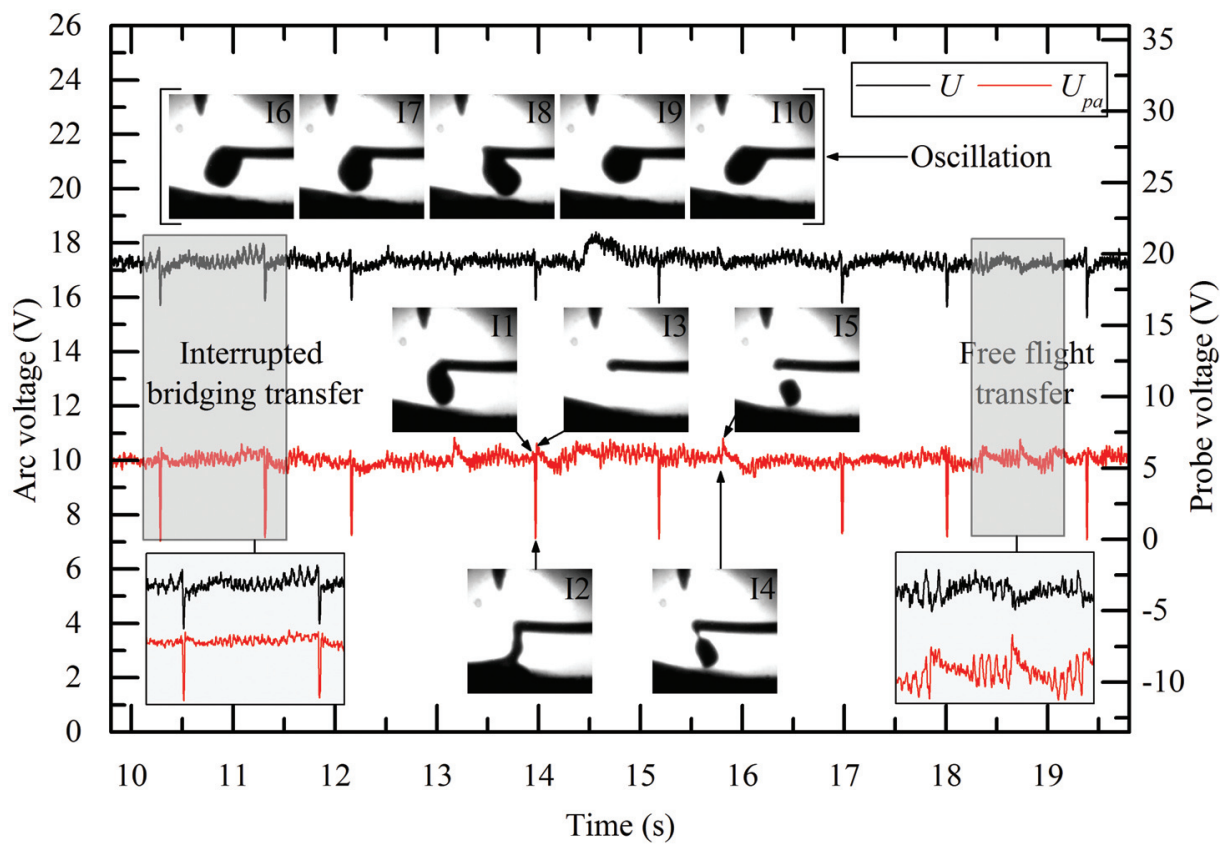

Fig. 5 - Electrical signals in Experiment \#2 (ETWD = $8.5 \mathrm{~mm}$ ).

stable during interrupted bridge transfer as it was during uninterrupted bridge transfer. From I2 to I3, the liquid bridge suddenly broke, contributing to a moderate increase in $U$ and a steep rise in $U_{p a}$, and it displayed the opposite rule for the process from I4 to I5. This is because the liquid bridge brought the anode closer to the cathode, which shorted the travel of the electron flow and made $U$ smaller.

The formation and breaking of the liquid bridge also caused the changes in both $U$ and $U_{p a}$, while its effect on $U_{p a}$ was more significant. In the practical welding process, it also caused a flickering arc and a series of crack sounds. When the filler wire was close to the workpiece, the interrupted bridging transfer with the above phenomenon easily to occurred at a fast metal transfer frequency.
In Fig. 5, the liquid metal is in a hybrid mode of interrupted bridging transfer (I1-I3) and free-flight transfer (I4-I5). It is conspicuous that the trends of voltage signals under the interrupted bridging transfer mode shown in Figs. 4 and 5 are different. The short-circuit liquid bridge only existed for a very short time during the bridging transfer at the ETWD in Fig. 5, which caused the voltage signals to stay at a higher value for a longer time and generate significant pulse-like fluctuations. The frequency of metal transfer was also lower, which reduced the frequency of the arc being periodically attracted and repelled by the filler wire. Moreover, the arc voltage, after each metal transfer, was almost lower than before each metal transfer because the liquid metal that originally occupied the local arc space was transferred to the weld pool and the influence of 


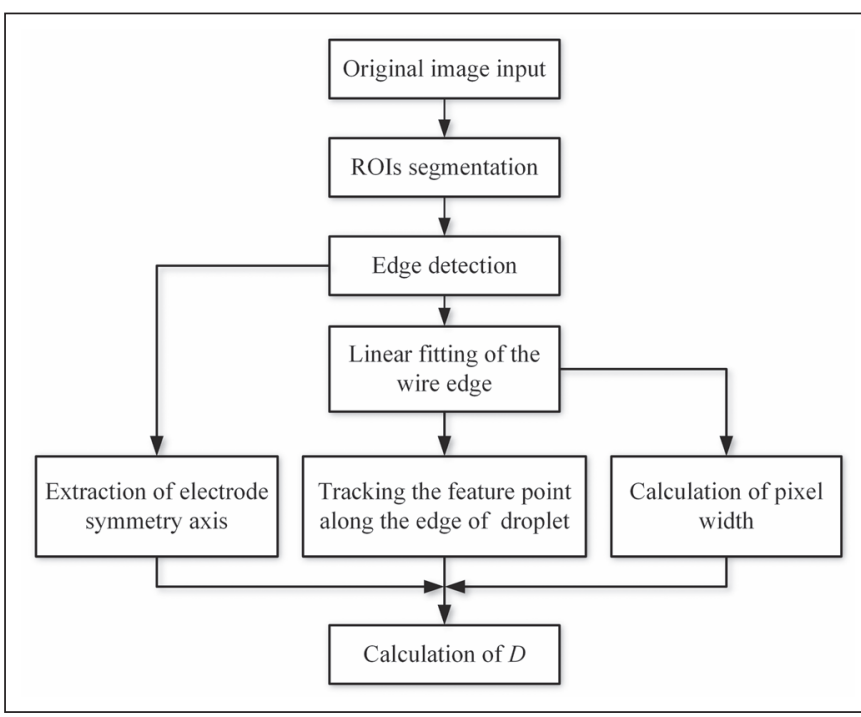

Fig. 6-Flow chart of the image processing algorithm.

the filler wire on the arc voltage was reduced. It could be inferred that the difference in arc voltage before and after the metal transfer was related to the amount of metal transferred each time. As for free-flight transfer, the arc voltage will only drop in moderation due to the absence of the liquid bridge. Furthermore, once the droplet is separated from the end of the filler wire, the distance between the filler wire and the weld pool will suddenly increase and the space occupied by the droplet will be released in a short time, so $U_{p a}$ will rise immediately. Besides, after metal transfer, the droplet will sometimes oscillate (e.g., I6-I10), which will cause diverse degrees of fluctuations in both $U$ and $U_{p a}$, just like the dynamic effects of the metal probe on the arc in the first part of the work (Ref. 6).

From the above, $U_{p a}$ could be used to conveniently monitor the status of the metal transfer. The information, such as the mode and frequency of metal transfer and the existence time of the short-circuit liquid bridge, could be more clearly reflected in $U_{p a}$ than in $U$.

\section{Characterization of Droplet Behavior}

As shown in Fig. 5, the liquid droplet can oscillate back and forth at the end of the filler wire before it enters the weld pool. To characterize this dynamic behavior, an image processing algorithm was developed to calculate the distance between the edge of the droplet and the symmetry axis of the tungsten electrode $(D)$. Its processing flow and schematic diagram are respectively shown in Figs. 6 and 7.

Figure 7 shows two regions of interest (ROIs), the tungsten electrode (ROI1) and the filler wire (ROI2), were segmented from the original image, and their edge points were extracted by a Sobel operator. For ROI1, the position of the symmetry axis of the electrode was obtained by calculating the average horizontal coordinate of electrode edge points. For ROI2, Hough transformation was used for linear fitting of the wire edges, and then the distance (number of pixels) between the two fitted line segments was calculated to obtain the actual width of one pixel based on the actual diameter of filler wire. To locate the feature point of the droplet edge closest to the symmetry axis of the electrode, one end-

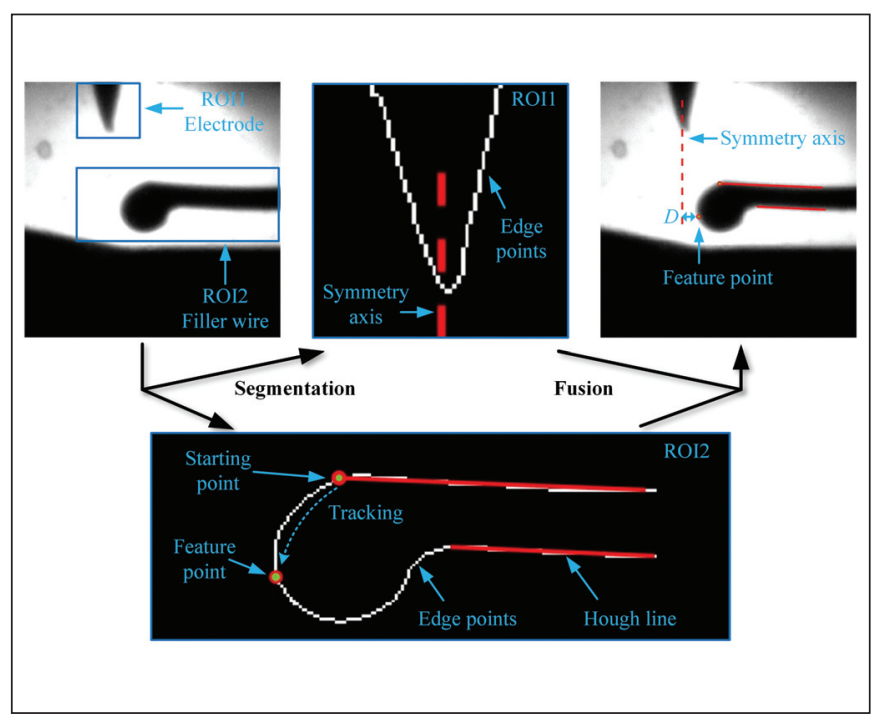

Fig. 7 - Schematic diagram of image processing.

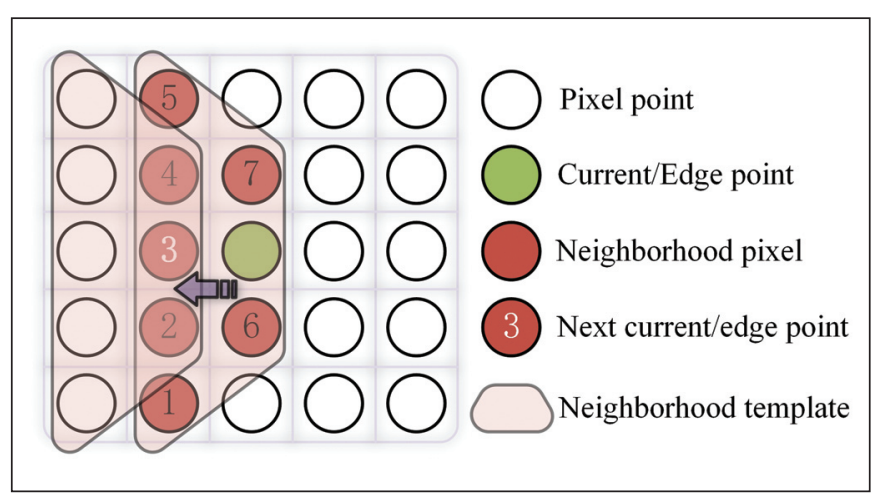

Fig. 8 - Schematic diagram of tracking the feature point.

point of the fitted line segment was selected as a starting point to track the feature point along the edge of the droplet (as demonstrated in ROI2 in Fig. 7). During the tracking procedure, if an edge point of the droplet was detected in the left neighborhood of the current point (as shown in Fig. 8), the detected point would become a new current point, otherwise the current point was the feature point. $D$ can be derived by calculating the distance from this feature point to the symmetry axis of the electrode, which can then be used to geometrically characterize the dynamic behavior of the liquid droplet in the time domain.

The behaviors of pendant droplets were studied based on Experiments \#3, \#4, and \#5, where different wire feed speeds were employed. Experimental results at different wire feed speeds are shown in Fig. 9. In Fig. 9A, within a period of time after the last metal transfer, the pendant droplet remaining at the end of the filler wire was too small to oscillate significantly (I1 and I2), and $D$ and $U$ kept the downward and upward trends, respectively, which showed that the end of the filler wire was getting closer and closer to the arc axis during this period. Once the liquid droplet contacted the weld pool, it quickly transferred from the filler wire to the weld pool under the influence of surface tension and gravity. Since the amount of filler wire melted in a short time was less than the amount of liquid 

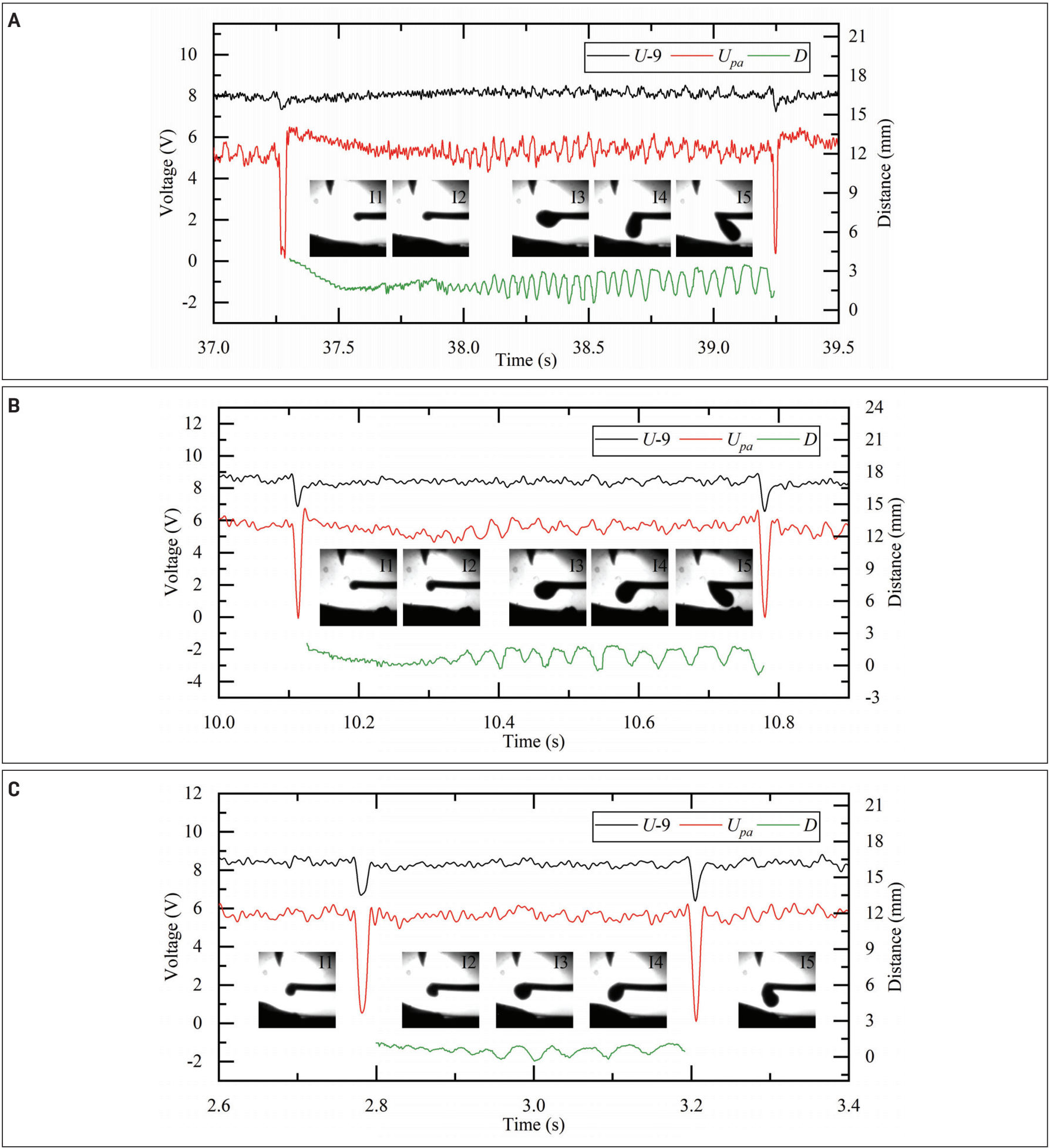

Fig. 9 - Experimental results at different wire feed speeds: $A$ - Experiment \#3 $\left(\mathrm{V}_{\mathrm{f}}=50.8 \mathrm{~cm} / \mathrm{min}\right) ; B-$ Experiment \#4 $\left(\mathrm{V}_{\mathrm{f}}=76.2\right.$ $\mathrm{cm} / \mathrm{min}) ; \mathrm{C}$ - Experiment \#5 $\left(\mathrm{V}_{\mathrm{f}}=101.6 \mathrm{~cm} / \mathrm{min}\right)$.

metal entering the weld pool, the end of the filler wire was far away from the symmetry axis of the tungsten electrode (as shown in I1) when the droplet transfer was just completed. Moreover, the further the filler wire was from the arc, the slower the melting rate, so the end of the filler wire approached the arc again at a decaying rate of move- ment (as shown in I2). Such a trend gradually became less pronounced with increasing wire feed speed, as shown in Fig. 9B and C.

As the melting rate of the filler wire gradually stabilized and the size of the droplet increased, the oscillation of the pendant droplet gradually became apparent. It can be ob- 


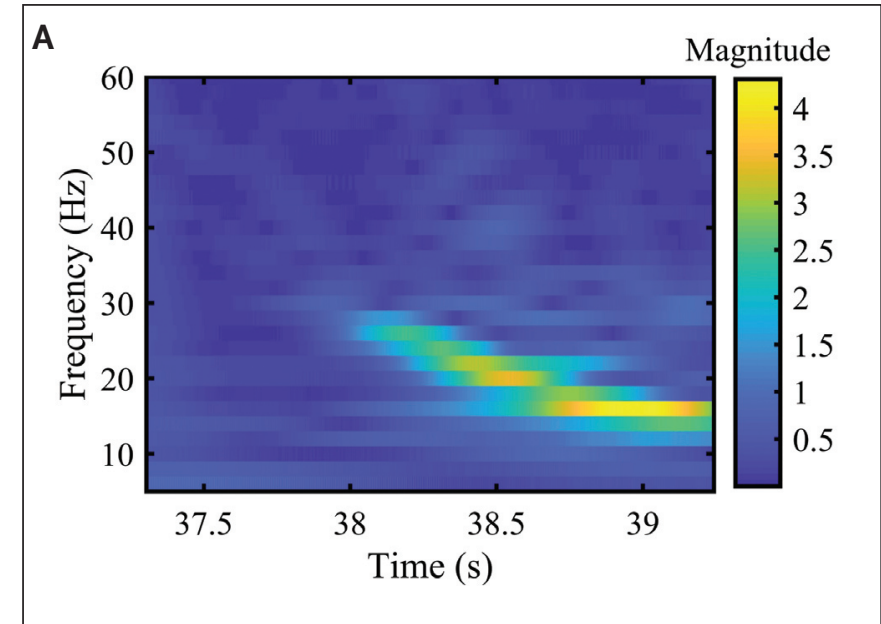

served in Fig. 9A that the curve of $D$ gradually changed from decreasing to fluctuating up and down after a metal transfer, which shows that the liquid droplet was gradually oscillating back and forth. This is because the liquid droplet has a free surface and it hangs in the arc space. Any random initial disturbance and uneven forces can easily trigger its oscillation. In Fig. 9A, I3 to I5 show the cases where a droplet with a volume oscillated around the end of the filler wire under the action of gravity and arc force as well as the blowing force of shielding gas, and its shape was constantly changing. As the wire feed speed increased, the metal transfer frequency increased, and because the droplet volume can only increase to a limited extent, its oscillation amplitude also decreased relatively, as presented in Fig. 9B and C.

A small enough droplet will not cause a large fluctuation in the voltage signals even if it oscillates. As the droplet grew, the area of the arc space it occupied gradually increased and its influence on the arc also increased, so the droplet oscillation could be reflected in the voltage signals. The droplet oscillation followed a similar mechanism to the dynamic disturbance of the arc space by the metal probe used in the first part of this work (Ref. 6), which also caused the voltage signals to oscillate regularly. If the wire feed speed is too slow, the end of the filler wire will be in the edge area of the arc, and then the droplet oscillation will cause a significant change in the probe voltage but will not be easily captured by the arc voltage, which also corresponds to an unstable melting of the filler wire. If the wire feed speed is too fast, its interference with the arc will be aggravated, which will be detrimental to the stability of the arc.

\section{Time-Frequency Characteristics of Droplet Oscillation}

To further clarify the frequency characteristics of the droplet oscillation and its effect on the electrical signals, the data of Experiments \#3, \#4, and \#5 (the data processed was taken between two bridge transfers) was converted from the time domain to the time-frequency domain for analysis using continuous wavelet transform. The selected mother wavelet was the complex Morlet wavelet with a bandwidth parameter of $3 \mathrm{~Hz}$ and a center frequency of $3 \mathrm{~Hz}$.

The results of continuous wavelet transform are shown in Figs. 10-12. It can be observed from the change of $D$ in Fig. 10
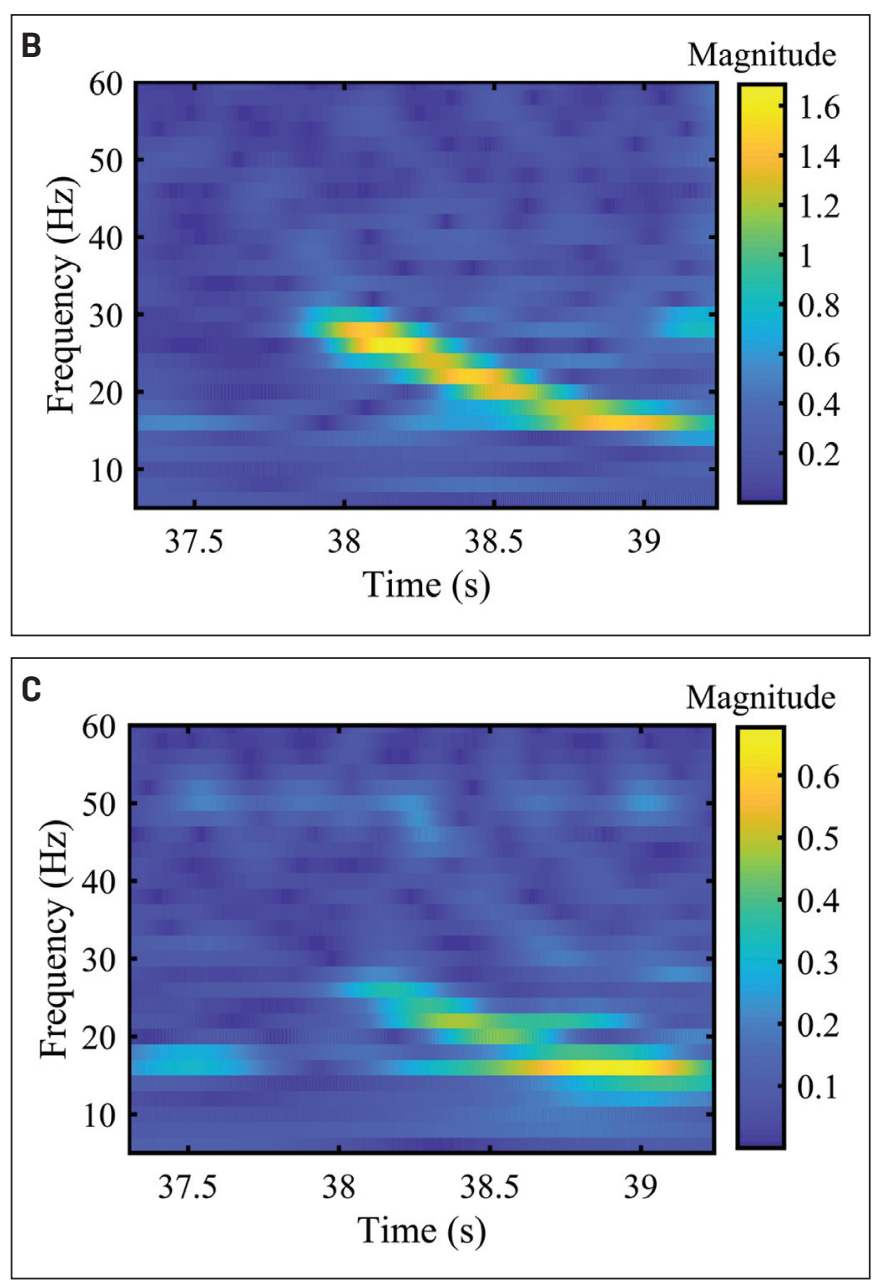

Fig. 10 - Spectrogram of continuous wavelet transform in EXperiment \#3 $\left(\mathrm{V}_{\mathrm{f}}=50.8 \mathrm{~cm} / \mathrm{min}\right): \mathrm{A}-\mathrm{D} ; \mathrm{B}-\mathrm{U}_{\mathrm{pa}} ; \mathrm{C}-\mathrm{U}$.

that as time increased, the oscillation frequency of the droplet had a continuous downward trend from about $30 \mathrm{~Hz}$ to less than $20 \mathrm{~Hz}$, and then remained stable. The same trend also appeared in $U$ and $U_{p a}$. As the wire feed speed increased, this continuous downward trend gradually became inconspicuous and the droplet oscillation frequency in the initial stage shifted to a lower frequency band, as presented in Fig. 11. When the wire feed speed was further increased, as shown in Fig. 12, the initial oscillation frequency of the droplet directly appeared at about $20 \mathrm{~Hz}$ and then remained relatively stable. It can be seen from the above analysis that the initial oscillation frequency was affected by the wire feed speed. This is because the increase in the wire feed speed brouht the droplet closer to the symmetry axis of the electrode, which weakened the asymmetry of the force on the left and right of the droplet. Although the droplet can oscillate at a lower frequency when the wire feed speed increases, its magnitude is not as large as the magnitude at the same oscillation frequency when the wire feed speed is smaller.

It can be further observed that no matter how the oscillation frequency of the droplet changes, the oscillation frequency of the voltage signals $U$ and $U_{p a}$ can follow the change, which means that the voltage signal can indeed be used as a characteristic electrical signal to describe the droplet oscilla- 

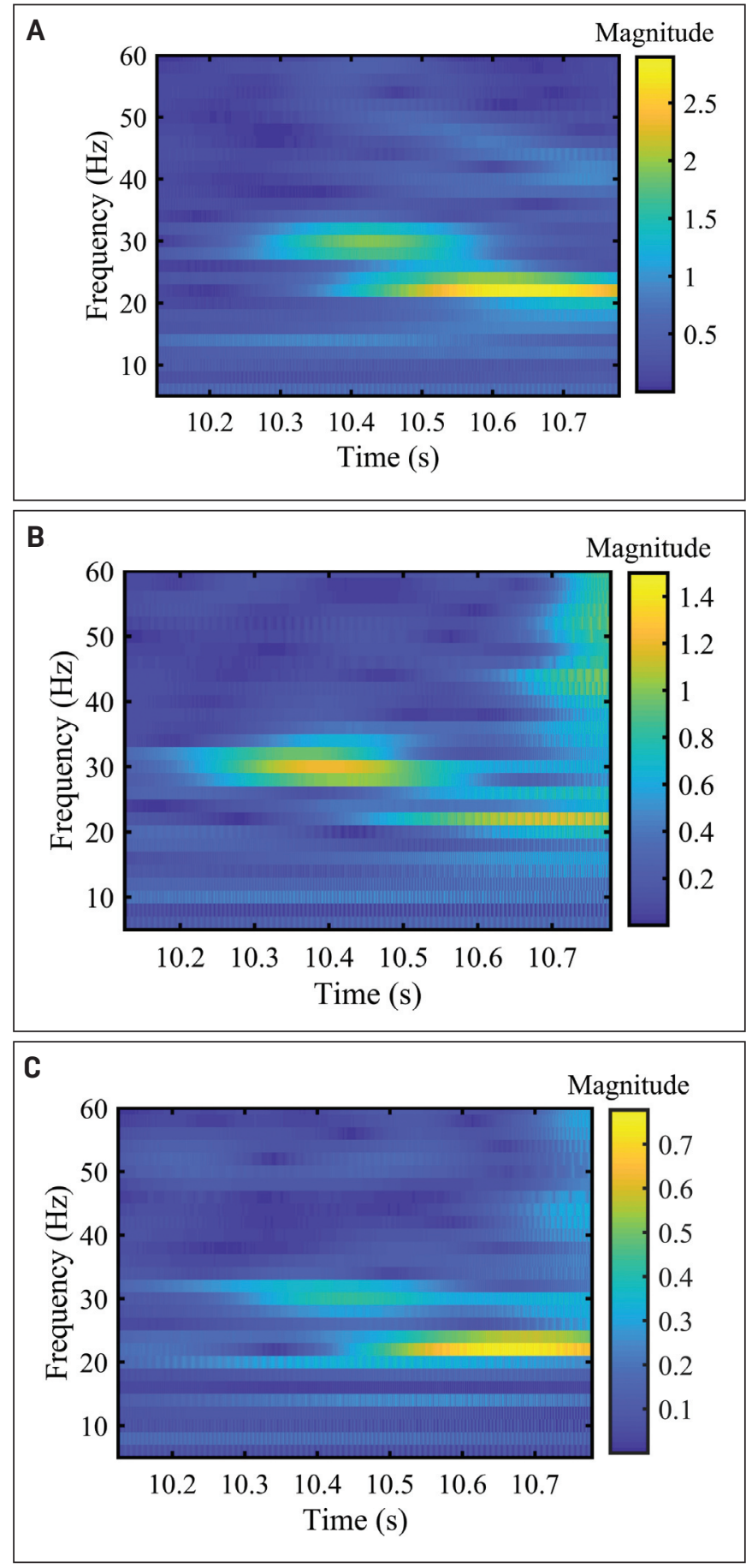

Fig. 11 - Spectrogram of continuous wavelet transform in EXperiment \#4 $\left(\mathrm{V}_{\mathrm{f}}=76.2 \mathrm{~cm} / \mathrm{min}\right): \mathrm{A}-\mathrm{D} ; \mathrm{B}-\mathrm{U}_{\mathrm{pa}} ; \mathrm{C}-\mathrm{U}$.

tion. Furthermore, the influence of droplet oscillation on $U_{p a}$ is greater than that on $U$ because the fluctuation magnitude of $U_{p a}$ was greater, and in the early stage of droplet oscillation, when $U$ had not noticeable fluctuations, $U_{p a}$ had been able to reflect the droplet oscillation. It can thus be concluded that $U_{p a}$ can more clearly characterize the droplet oscillation than $U$, and when the droplet size is not large enough, it can detect droplet oscillation more sensitively than $U$. Therefore, $U_{p a}$ can be considered for use in monitoring droplet oscillation.
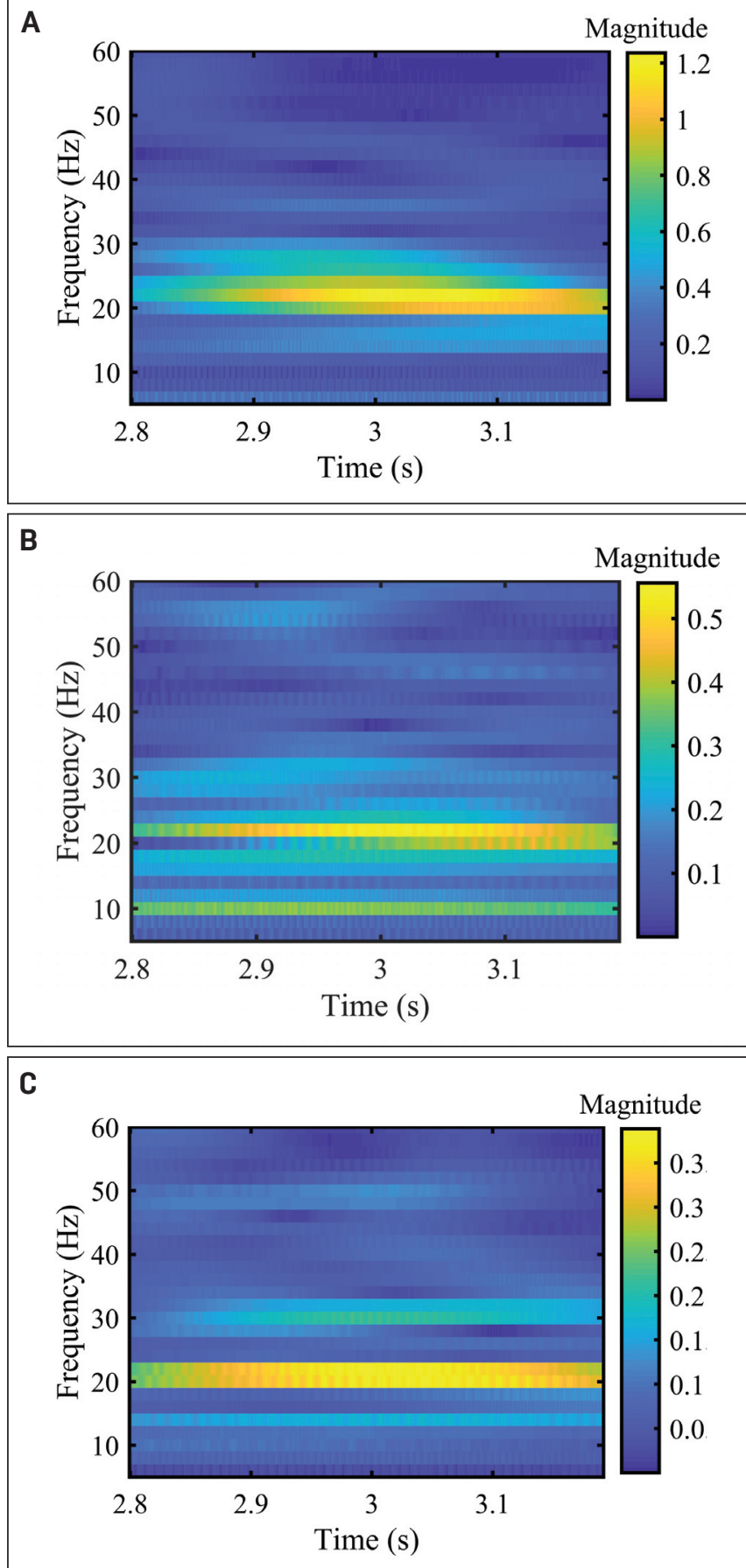

Fig. 12 - Spectrogram of continuous wavelet transform in EXperiment \#5 $\left(\mathrm{V}_{\mathrm{f}}=101.6 \mathrm{~cm} / \mathrm{min}\right): \mathrm{A}-\mathrm{D} ; \mathrm{B}-\mathrm{U}_{\mathrm{pa}} ; \mathrm{C}-\mathrm{U}$.

\section{Conclusions}

This part of the work analyzed the dynamic behaviors of droplets in cold-wire GTAW based on the proposed local arcsensing method of detecting probe voltage. The following main conclusions can be drawn:

1) The dynamic behaviors of the droplets affecting the GTA can be roughly divided into the following: the droplet transfer to the weld pool, occupying the original arc space with differ- 
ent volumes, and the dynamic oscillation in different areas of the arc column. Information about these dynamic behaviors can be easily reflected in the probe voltage $\left(U_{p a}\right)$.

2) For the bridging transfer, the formation and breaking of the liquid bridge will cause the changes in both $U_{p a}$ and arc voltage $(U)$, while its effect on $U_{p a}$ is more significant. For free-flight transfer, once the droplet is separated from the filler wire, $U_{p a}$ will also respond immediately. Thus, $U_{p a}$ can be used to conveniently monitor the metal transfer.

3) Due to the asymmetrical force, the droplet is liable to oscillate, and will cause the voltage signals to oscillate after having a volume. The increase in the wire feed speed will bring the droplet closer to the arc axis and weaken the asymmetry of the force, thus tending to reduce the initial oscillation frequency. Besides, $U_{p a}$ can more clearly characterize the droplet oscillation than $U$.

In the practical GTAW process, a stable and reasonable metal transfer may not always be guaranteed because of the dynamic time variation of this process and other random factors, such as unstable wire feeding, the subsidence of the weld pool during the welding of butt joints, and the variation of the deposited layer height caused by heat accumulation during GTA-based additive manufacturing. Thus, it makes sense to introduce in-process monitoring and even feedback control of quality stability. In light of this, the probe voltage may be a good indicator of characterizing the droplet behaviors and would be a candidate signal to monitor the process stability. From this perspective, the sensing method proposed in this work paves the way for real-time control of the weld quality for joining, such as orbital welding or welding in all positions, as well as the quality of GTAbased additive manufacturing.

\section{Acknowledgments}

This study is supported by the National Natural Science Foundation of China (Grant No.: 51505326), Natural Science Foundation of Tianjin (Grant No.: 16JCQNJC04300), and Regional Demonstration Project of Marine Economic Innovation and Development (Grant No.: BHSF2017-10).

\section{References}

1. Huang, Y. M., Wu, D., Zhang, Z. F., Chen, H. B., and Chen, S. B. 2017. EMD-based pulsed TIG welding process porosity defect detection and defect diagnosis using GA-SVM. Journal of Materials Processing Technology 239: 92-102. DOI: 10.1016/j.jmatprotec.2016.07.015

2. Chokkalingham, S., Chandrasekhar, N., and Vasudevan, M. 2012. Predicting weld bead width and depth of penetration from infrared thermal image of weld pool using artificial neural network. Journal of Intelligent Manufacturing 23(5): 1995-2001. DOI: 10.1784/insi.2012.54.5.272

3. Wang, H., and Kovacevic, R. 2002. Feasibility study of acoustic sensing for the welding pool mode in variable-polarity plasma arc welding. Proceeding of the Institute of Mechanical Engineers, Part B. Journal of Engineering Manufacture 216(10): 1355-1366. DOI:10.1243/095440502320405449

4. Wang, Z. J., Zhang, Y. M., and Wu, L. 2010. Measurement and estimation of weld pool surface depth and weld penetration in pulsed gas metal arc welding. Welding Journal 89(6): 117-s to 126-s.

5. Chen, J. S., Chen, J., Zhang, K., Feng, Z., and Zhang, Y. M. 2018. Dynamic reflection behaviors of weld pool surface in pulsed GTAW. Welding Journal 97(6): 191-s to 206-s. DOI: 10.29391/ 2018.97.017

6. Zou, S. Y., Wang, Z. J., Hu, S. S., Zhao, G. C., Wang, W. D., and Chen, Y. Q. 2020. Effects of filler wire intervention on gas tungsten arc: Part I - Mechanism. Welding Journal 99(9): 246-s to 254-s. DOI: $10.29391 / 2020.99 .023$

SHUANGYANG ZOU, ZHIJIANG WANG (wangzj@tju.edu.cn), SHENGSUN HU, GUANCHENG ZHAO, and WANDONG WANG are with the Tianjin Key Laboratory of Advanced Joining Technology, School of Materials Science and Engineering, Tianjin University, Tianjin, China. YOUQUAN CHEN is with the Department of Mechanical Engineering, College of Humanities \& Information, Changchun University of Technology, Changchun, China.

\section{Authors: Submit Research Papers Online}

Peer review of research papers is now managed through an online system using Editorial Manager software. Papers can be submitted into the system directly from the Welding Journal page on the AWS website (aws.org) by clicking on "submit papers." You can also access the new site directly at editorialmanager.com/wj/. Follow the instructions to register or log in. This online system streamlines the review process, and makes it easier to submit papers and track their progress. By publishing in the Welding Journal, more than 70,000 members will receive the results of your research.

Additionally, your full paper is posted on the American Welding Society website for FREE access around the globe. There are no page charges, and articles are published in full color. By far, the most people, at the least cost, will recognize your research when you publish in the world-respected Welding Journal. 